\title{
Structure and Cathodoluminescence of $\mathrm{Y}_{2} \mathrm{O}_{3}: \mathrm{Eu}$ Thin Films obtained at Different Conditions
}

\author{
I.O. Bordun, O.M. Bordun, I.Yo. KukharskyY* and Zh.Ya. Tsapovska \\ Ivan Franko Lviv National University, Dragomanova Str. 50, 79005, Lviv, Ukraine
}

\begin{abstract}
The structure of the surface and spectra of the cathodoluminescence of $\mathrm{Y}_{2} \mathrm{O}_{3}$ :Eu thin films when changing the activator concentration in the range 1.0-7.5\% by mole obtained by RF sputtering were investigated. On the base of the shape of the cathodoluminescence spectra at different concentrations of the activator, the possibility of irregular solutions creating of yttrium and europium oxides and the structural features of the small and large crystallites forming the film $\mathrm{Y}_{2} \mathrm{O}_{3}: \mathrm{Eu}$ is shown. The dependence of the cathodoluminescence intensity on the energy of the exited electrons and current density of electron irradiation was established.
\end{abstract}

DOI: 10.12693/APhysPolA.133.914

PACS/topics: 68.37.Ps, 78.60.Hk, 81.15.-Z

\section{Introduction}

Among the large number of materials the luminescent materials have particular importance for using in the optoelectronics. They are used for creating displays, scintillators, for the means of information recording and its visualization. The cubic $\mathrm{Y}_{2} \mathrm{O}_{3}$ yttrium oxide activated with rare earth ions is one of the widespread crystalline matrix of phosphors. Among them the most investigated and widely used phosphor of micron dispersion is the $\mathrm{Y}_{2} \mathrm{O}_{3}$ :Eu one with red emission region [1-3]. The disadvantage of this material is unsatisfactory morphology of the particles that do not ensure a smooth and uniform coating of the screens and high resolution. Such situation has led to active investigation of various thin film and nanostructure objects based on $\mathrm{Y}_{2} \mathrm{O}_{3}: \mathrm{Eu}$ [4-7].

It has been observed that the number of properties of this material changes when the size of crystals reaches nanometer dimensions. These changes can be caused both by quantum-size effects and by the increasing role of various surface effects. The combination of small sizes of crystalline particles and the presence of the dopant - the center of luminescence - $\mathrm{Eu}^{3+}$ ion provides the uniform coverage of the screen at deposition of $\mathrm{Y}_{2} \mathrm{O}_{3}: \mathrm{Eu}$ thin films which consist of nanocrystalline grains, and thus provides the efficiency and stability of luminescence and promotes the expansion of potential applications. It should be noted that a number of methods are used to obtain films based on $\mathrm{Y}_{2} \mathrm{O}_{3}$. Such films differ in their optical and luminescence properties due to their various perfection. Therefore, in this paper the structure of surface, the spectral properties and the efficiency of CL glow of $\mathrm{Y}_{2} \mathrm{O}_{3}: \mathrm{Eu}^{3+}$ thin films, obtained by radio-frequency ionplasmous sputtering, depending on the concentration of the dopant and the size of the crystallites are studied.

\footnotetext{
* corresponding author; e-mail: kukharskij@electronics.lnu.edu.ua
}

It should be noted that method of radio-frequency ionplasmous sputtering is optimal for obtaining of the most homogeneous semiconductor and dielectric films $[8,9]$.

\section{Experimental technique}

Thin films of $\mathrm{Y}_{2} \mathrm{O}_{3}$ :Eu with $0.2-1.0 \mu \mathrm{m}$ thickness were obtained by radio-frequency ion-plasmous sputtering in $100 \%$ atmosphere of oxygen or in $100 \%$ atmosphere of argon on amorphous fused quartz $v-\mathrm{SiO}_{2}$ substrates. The activator concentration varied from 1.0 to $7.5 \mathrm{~mol} . \%$. After thin films deposition the heat treatment was conducted in air in $950-1050{ }^{\circ} \mathrm{C}$ temperature region. The $\mathrm{X}$-ray diffraction investigations showed the presence of the polycrystalline films structure preferentially oriented in the (222) plane. The form of the obtained diffraction patterns is almost similar to the diffraction patterns of pure $\mathrm{Y}_{2} \mathrm{O}_{3}$ films, which we presented in [10]. In this case, in the $\mathrm{Y}_{2} \mathrm{O}_{3}$ :Eu films obtained under an argon atmosphere, the reflex from the (440) plane has a somewhat higher intensity.

The morphology of the surface of the films was investigated by an atomic force microscope (AFM) Solver P47 PRO. The processing of the experimental data and the computation of the parameters of the surface morphology were carried out using the Image Analysis 2 software package.

The investigation of CL properties was carried out in the regime of pulsed electronic excitation. The luminescence spectra were recorded on a device that was mounted on the basis of the MDR-12 spectrophotometer. The measured signal was transmitted via an analog-todigital interface converter to an IBM/PC computer for the luminescence spectrum recording. The scanning of the wavelengths by a monochromator was carried out by the instrumentality of a stepping motor, which was controlled by a computer through a control unit. The luminescent spectra were investigated in the range from 200 to $800 \mathrm{~nm}$ at $295 \mathrm{~K}$. 


\section{Results and discussion}

Figures 1 and 2 show the typical micrographs of the $\mathrm{Y}_{2} \mathrm{O}_{3}$ :Eu films' surface with different concentrations of activator obtained by AFM. As can be seen from the obtained results, the concentration of the activator $\mathrm{Eu}^{3+}$ has a considerable influence on the sizes of the crystalline grains and the surface films roughness. Moreover, even the method of obtaining is less influential on these parameters of the films structure (Fig. 1). The characteristic structural parameters of the $\mathrm{Y}_{2} \mathrm{O}_{3}$ :Eu films' surface are given in Table I.

TABLE I

The surface roughness of the films, the average grains' sizes and the areas of crystallite in $\mathrm{Y}_{2} \mathrm{O}_{3}$ :Eu thin films. $\mathrm{A}$ - the concentration of $\mathrm{Eu}^{3+}$ activator [mol.\%], B Method of obtaining, C - The surface roughness [nm], $\mathrm{D}$ - The average grain size $[\mathrm{nm}], \mathrm{E}$ - The average area of crystallites $\left[\mathrm{nm}^{2}\right]$

\begin{tabular}{c|c|c|c|c}
\hline \hline $\mathrm{A}$ & $\mathrm{B}$ & $\mathrm{C}$ & $\mathrm{D}$ & $\mathrm{E}$ \\
\hline 1.0 & Discrete evaporation & 5.65 & 15.68 & 242 \\
\hline 1.0 & RF sputtering & 5.31 & 15.86 & 307 \\
\hline 2.5 & RF sputtering & 17.62 & 43.14 & 8027 \\
\hline 5.0 & RF sputtering & 81.33 & 352.94 & 111157
\end{tabular}

It is characteristic that $\mathrm{Y}_{2} \mathrm{O}_{3}: \mathrm{Eu}$ films obtained by radio-frequency ion-plasmous sputtering have somewhat larger sizes of the crystalline grains than films obtained by discrete sputtering, although they have a lower surface roughness. At the same time, an increase in the concentration of the $\mathrm{Eu}^{3+}$ activator leads to a substantial increase in both the crystallite sizes and the roughness of the $\mathrm{Y}_{2} \mathrm{O}_{3}$ :Eu films surface relief (Fig. 2).

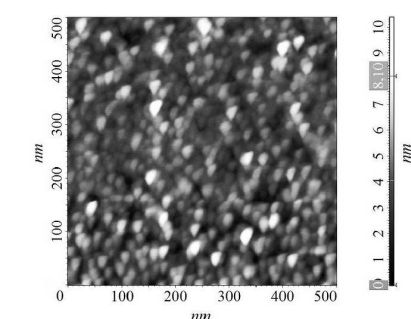

a

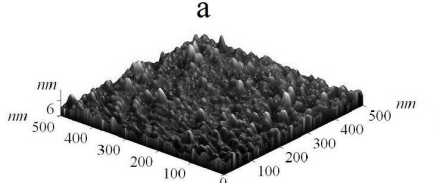

b
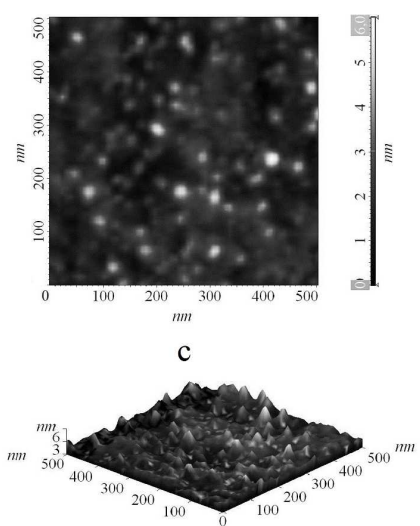

d
Fig. 1. The image of surface morphology of $\mathrm{Y}_{2} \mathrm{O}_{3}: \mathrm{Eu}$ thin films obtained by the method of discrete evaporation ((a), (b)) and RF ion-plasmous sputtering in argon atmosphere ((c), (d)) with concentration of activator 1 mol.\%. The images (a) and (c) are two-dimensional, (b) and (d) are three-dimensional.

The investigation of the CL spectra of $\mathrm{Y}_{2} \mathrm{O}_{3}:$ Eu thin films with different concentrations of the activator shows that the shape of the spectra does not change, at least

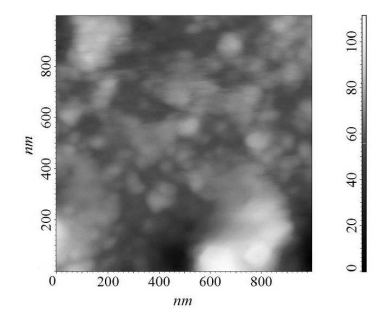

a

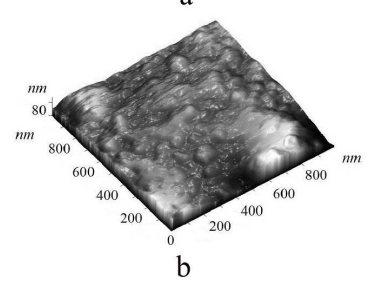

b

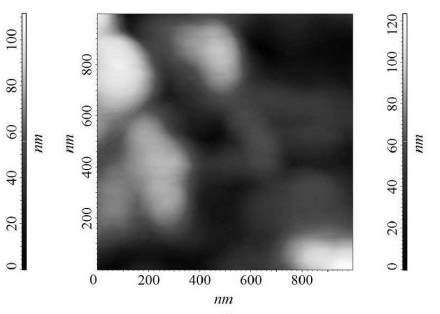

${ }_{\mathrm{c}}^{\mathrm{nm}}$

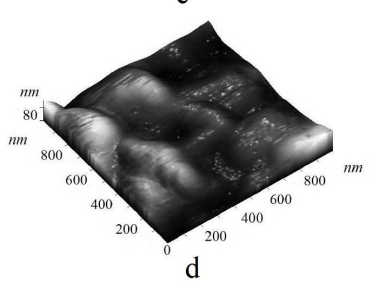

Fig. 2. The image of surface morphology of $\mathrm{Y}_{2} \mathrm{O}_{3}: \mathrm{Eu}$ thin films obtained by the method of RF ion-plasmous sputtering in argon atmosphere with concentration of activator $2.5 \mathrm{~mol} \%$ ((a), (b)) and $5 \mathrm{~mol} \%$ ((c), (d)). The images (a) and (c) are two-dimensional, (b) and (d) are three-dimensional.

when the concentration of the activator varies within the range from 1.0 to $7.5 \mathrm{~mol} \%$. Figure 3 shows the characteristic CL spectra of $\mathrm{Y}_{2} \mathrm{O}_{3}$ :Eu films, obtained at different concentrations of the activator. As can be seen from the figure, the narrow bands of luminescence due to intracenter transitions between the electron shells of the $\mathrm{Eu}^{3+}$ activator appear in the spectra. These bands are associated with the allowed ${ }^{5} D_{0}{ }^{-} F_{1}$ magnetic dipole transitions (for $\mathrm{Eu}^{3+}$ ions in $C_{2}$ and $C_{3 i}$ sites of $\mathrm{Y}_{2} \mathrm{O}_{3}: \mathrm{Eu}$ crystal lattice) and the allowed ${ }^{5} \mathrm{D}_{0}{ }^{7} F_{2}$ electric dipole transitions (for $\mathrm{Eu}^{3+}$ ions only in $C_{2}$ sites) $[11,12]$. The luminescence in the band with maximum at $612 \mathrm{~nm}$ is determined by the ${ }^{5} D_{0^{-}}{ }^{7} F_{2}$ electric dipole transition, which is very sensitive to the nearest environment of the radiating $\mathrm{Eu}^{3+}$ ion. The band with maximum at $596 \mathrm{~nm}$ is determined by the ${ }^{5} D_{0^{-}}{ }^{7} F_{1}$ magnetic dipole transition, which is insensitive to the nearest environment [13-16].

In addition, it was found that $\mathrm{Y}_{2} \mathrm{O}_{3}: \mathrm{Eu}$ films deposited in the atmosphere of both argon and oxygen have a maximum yield of CL at the concentration of activator of 5.0 mol.\% (Fig. 3, insert). At lower concentrations the sizes of the crystalline grains decrease that leads to increase of the relative contribution of grain-boundary scattering. The most likely, at high concentrations of the activator the concentration quenching is observed due to the creation of a more defective structure and the increase in the number of nonradiative relaxation channels. The results obtained can be explained by assuming that the increase in the concentration of the activator can also lead to increase of the $\mathrm{Eu}^{3+}$ ions' diffusion into the sites of $\mathrm{Y}_{2} \mathrm{O}_{3}$ crystal lattice with $C_{3 i}$ point symmetry, for which the electric dipole radiation is forbidden according to the selection rules.

Our results agree with the results of $\mathrm{Y}_{2} \mathrm{O}_{3}$ :Eu nanoparticles studies conducted by $[3,17,18]$ authors. These re- 


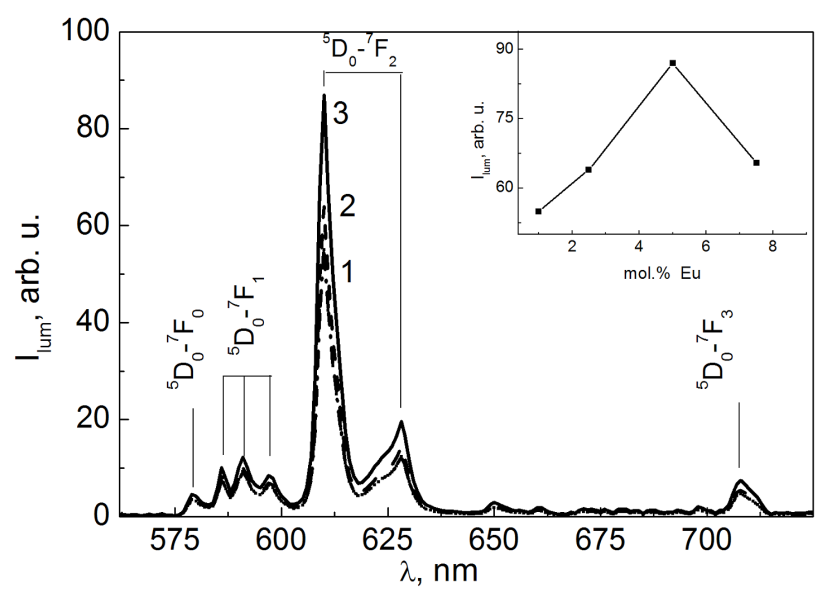

Fig. 3. The CL spectra of $\mathrm{Y}_{2} \mathrm{O}_{3}$ :Eu thin films obtained by radio-frequency ion plasma sputtering in argon atmosphere at the concentration of activator of $1 \mathrm{~mol} \%$ (1), $2.5 \mathrm{~mol} . \%$ (2), and $5 \mathrm{~mol} \%$ (3). The parameters of electron irradiation pulses: the current density of the electron beam is $j=5 \times 10^{-2} \mathrm{~A} / \mathrm{m}^{2}$, the duration of pulse is $5 \times 10^{-4} \mathrm{~s}$, the gap between pulses $0.1 \mathrm{~s}$, the energy of the excited electrons is $5 \mathrm{keV}$. In the inset, the dependence of the intensity of the ${ }^{5} D_{0}-{ }^{7} F_{2}$ transition for the CL spectra of $\mathrm{Y}_{2} \mathrm{O}_{3}: \mathrm{Eu}$ thin films from the concentration of activator.

sults indicate that due to increases of the activator concentration the increase of the crystalline grain sizes of the $\mathrm{Y}_{2} \mathrm{O}_{3}: \mathrm{Eu}$ film occurs as well as for the luminescence of $\mathrm{CL}$ the relative contribution of $\mathrm{Eu}^{3+}$ ions emission at sites with $C_{2}$ point symmetry increases relatively to the sites with $C_{3 i}$ point symmetry (see Fig. 3 ). Such a deviation most likely indicates on the possibility for creation of yttrium and europium oxide irregular solutions in $\mathrm{Y}_{2} \mathrm{O}_{3}$ :Eu thin film during its deposition and annealing. The formation of irregular solutions of $\mathrm{Y}$ and $\mathrm{Eu}$ oxides during the synthesis of sub- $\mu$ m phosphorus $\mathrm{Y}_{2} \mathrm{O}_{3}$ :Eu by a sol-gel method is also indicated in [17]. The increase in the relative amount of $\mathrm{Eu}^{3+}$ ions in the $C_{2}$ position in larger crystallites is most likely due to a decrease in the local symmetry of the europium ions environment by oxygen ions. Since the films were annealed in air, during the annealing process, the local symmetry of the $\mathrm{Eu}^{3+}$ ions increased primarily near the surface. It is clear that such near-surface effects are more noticeable in smaller crystallites, since their relative contribution will be more significant in the observed luminescence spectra.

The investigation of the CL intensity dependence on the energy of the excitation electrons was carried out at different pulse durations of electron irradiation. The typical dependences for the obtained films are shown in Fig. 4a.

According to Fig. 4a, the intensity of CL increases with the excitation energy increase. Such a course of this dependence for $\mathrm{Y}_{2} \mathrm{O}_{3}$ : Eu films is observed up to a certain value, which is determined by the thickness of the film In particular, for $\mathrm{Y}_{2} \mathrm{O}_{3}: \mathrm{Eu}$ film with thickness of $0.20 \mu \mathrm{m}$, as can be seen from Fig. 3a, this energy is in the region of
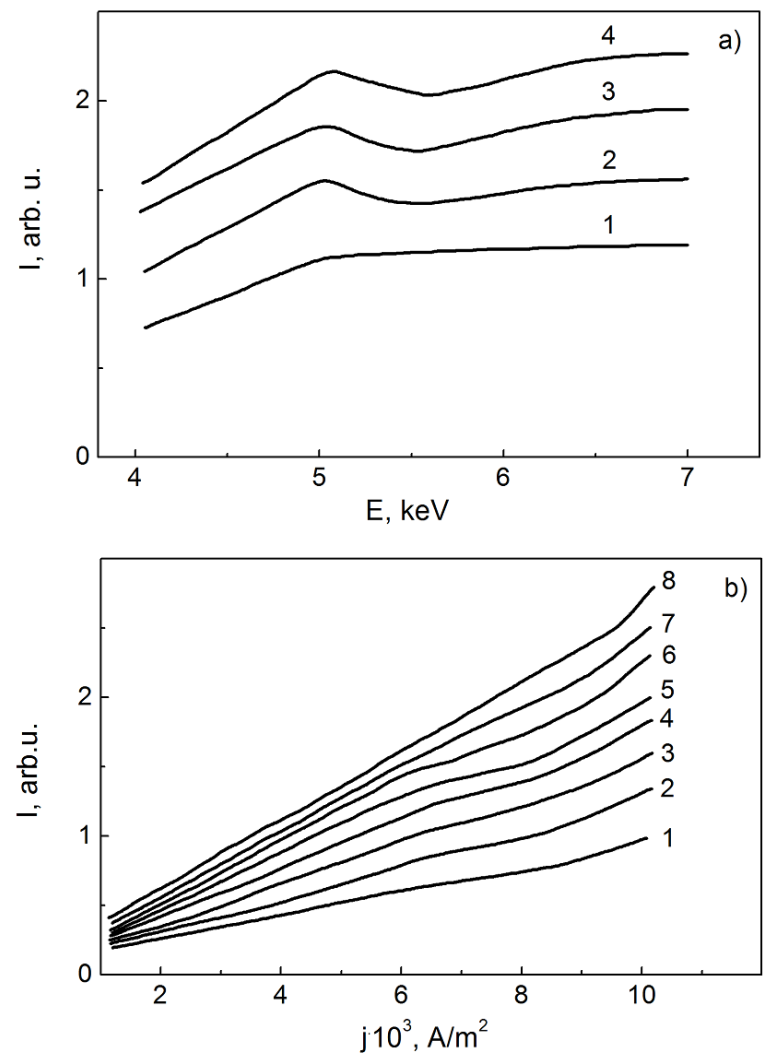

Fig. 4. The dependence of the intensity of CL of $\mathrm{Y}_{2} \mathrm{O}_{3}: \mathrm{Eu}$ thin films from the energy of the excited electrons (a) and the current density of the electron excitation (b). The concentration of activator is $2.5 \%$ (a) and $5 \%(\mathrm{~b})$, the deposition atmosphere is $\operatorname{Ar}(100 \%)$. The parameters of electron irradiation pulses: (a) the current density of the electron beam is $10^{-2} \mathrm{~A} / \mathrm{m}^{2}$, the duration of excitation pulses $4 \times 10^{-4} \mathrm{~s}(1), 6 \times 10^{-4} \mathrm{~s}(2)$, $8 \times 10^{-4} \mathrm{~s}(3), 10^{-3} \mathrm{~s}(4)$; (b) the energy of the excited electrons is $2.5 \mathrm{keV}$, the duration of pulse is $3 \times 10^{-4} \mathrm{~s}$ (1), $4 \times 10^{-4} \mathrm{~s}(2), 5 \times 10^{-4} \mathrm{~s}(3), 6 \times 10^{-4} \mathrm{~s}(4), 7 \times 10^{-4} \mathrm{~s}$ (5), $8 \times 10^{-4} \mathrm{~s}(6), 9 \times 10^{-4} \mathrm{~s}(7), 10^{-3} \mathrm{~s}(8)$. The gap between pulses is $0.1 \mathrm{~s}$.

$5.0 \mathrm{keV}$. After that the intensity of the CL is somewhat reduced and when the excitation energy reaches $5.5 \mathrm{keV}$ the intensity again begins to grow. Such a course of this dependence is apparently due to the fact that the excited beam begins to pass through the film and reaches the substrate when the excitation electrons reach energy of $5.0 \mathrm{keV}$. As a result, the electrons are redistributed between the film and the substrate that leads to decrease the intensity of the CL in the range from 5.0 to $5.5 \mathrm{keV}$. It is clear that the value of $5.5 \mathrm{keV}$ is not determinative for the $\mathrm{Y}_{2} \mathrm{O}_{3}: \mathrm{Eu}$ material, but is determined by the thickness of the deposited film [19]. At decrease (increase) of the film thickness the electrons energy at which the maximum intensity of the radiation is reached, will be decreased (increased).

It should be noted that the intensity of CL is a complex function of the excitation energy and radiation dose. 
Since the measurements (Fig. 4a) were carried out at fixed number of excitation electrons, it is clear that a further increase of the CL intensity at the excitation energy above $5.5 \mathrm{keV}$ can be explained only by the energy increase of the excitation electrons. When the duration of the exciting pulse was $4 \times 10^{-4}$ s, i.e. at the minimum dose of irradiation in this investigation, the abovedescribed minimum of the CL intensity was not observed.

With the help of this approach, one can also explain a rather sharp increase of the CL intensity with the increase of the exciting beam current (Fig. 4b). Analyzing the mechanism of the CL excitation of $\mathrm{Y}_{2} \mathrm{O}_{3}: \mathrm{Eu}$ phosphor, one must start from the fact that when the concentration of excitation particles increases then the intensity of CL must increase directly proportional. When the current of excitation reaches a certain critical value, the growth of intensity should slow down and reach saturation, since all possible luminescence centers begin to participate in process of CL. However, the experimental dependence (Fig. 4b) somewhat differs from directly proportional. The intensity of CL increases somewhat slower. This allows us to conclude that in this cathodophosphor the excitation energy is not completely transferred from the exciting electron to the radiation center, i.e. the part of the energy goes to nonradiative processes, that could be caused by a degree of the structural inhomogeneity of the films. At the same time, with increase of the excitation time, i.e. when the dose of irradiation is increased, the form of this dependence increasingly approaches to directly proportional.

\section{Conclusions}

The conducted investigations show that at radiofrequency ion-plasmous sputtering of $\mathrm{Y}_{2} \mathrm{O}_{3}$ : Eu thin films the increase of the activator concentration in the range from 1.0 to $5.0 \mathrm{~mol} \%$ leads to a significant increase of the crystallites size, which form the film from 15.86 to $352.94 \mathrm{~nm}$, respectively. The maximum yield of CL for the investigated films deposited in argon atmosphere and in oxygen atmosphere is observed at the concentration of activator of $5 \mathrm{~mol} . \%$. On the basis of the analysis of the CL spectra shape, it is established that when the concentration of the activator increases in the luminescence of $\mathrm{CL}$, the relative contribution of the $\mathrm{Eu}^{3+}$ ions' emission at the sites of the $\mathrm{Y}_{2} \mathrm{O}_{3}$ crystal lattice with $C_{2}$ point symmetry relatively to the sites with $C_{3 i}$ point symmetry increases. The assumption is made that in $\mathrm{Y}_{2} \mathrm{O}_{3}: \mathrm{Eu}$ thin films the excitation energy is not completely transferred from the exciting electron to the center of radiation, and some of the energy goes to nonradiative processes.
The improvement in the transfer of energy from the electrons absorbed by the film to the activator centers is observed with an increase in the irradiation dose, i.e. the duration of the CL exciting pulses from $3 \times 10^{-4} \mathrm{~s}$ to $10^{-3} \mathrm{~s}$.

\section{References}

[1] N. Yamamoto, Cathodoluminescence, InTech, Croatia 2012.

[2] A.S. Bugaev, V.B. Kireev, E.P. Sheshin, A.Yu. Kolodyazhn, Phys.-Usp. 58, 792 (2015).

[3] S.H. Cho, S.H. Know, J.S. Yoo, C.W. Oh, J.D. Lee, K.J. Hong, S.J. Kwone, J. Electrochem. Soc. 147, $3143(2000)$.

[4] Q. Dai, M.E. Foley, C.J. Breshike, A. Lita, G.F. Strouse, J. Am. Chem. Soc. 133, 15475 (2011).

[5] C. Shanga, X. Shang, Y. Qu, M. Li, Chem. Phys. Lett. 501, 480 (2011).

[6] R. Srinivasan, N.R. Yogamalar, J. Elanchezhiyan, R.J. Joseyphus, A.C. Bose, J. Alloys Comp. 496, $472(2010)$.

[7] P. Packiyaraj, P. Thangadurai, J. Lumin. 145, 997 (2014).

[8] H. Frey, H.R. Khan, Handbook of Thin Film Technology, Springer, Berlin 2015.

[9] E.V. Berlin, L.A. Seidman, Ion-Plasma Processes in Thin-Film Technology, Technosphere, Moscow 2010 (in Russian).

[10] O.M. Bordun, I.O. Bordun, I.Y. Kukharskyy, J. Appl. Spectrosc. 82, 390 (2015).

[11] N.C. Chang, J.B. Gruber, J. Chem. Phys. 41, 3227 (1964).

[12] G. Blasse, B.C. Grabmaier, Luminescent Materials, Springer, Berlin 1994.

[13] H. Shi, X.-Y. Zhang, W.-L. Dong, X.-Y. Mi, N.-L. Wang, Y. Li, H.-W. Liu, Chin. Phys. B 25, 047802 (2016).

[14] S. Som, S.K. Sharma, S.P. Lochab, Mater. Res. Bull. 48, 844 (2013).

[15] F.C. Romo, A.G. Murillo, D.L. Torres, N.C. Castro, V.H. Romero, E. Rosa, V.G. Febles, M.G. Hernández, Opt. Mater. 32, 1471 (2010).

[16] G.S. Gowd, M.K. Patra, S. Songara, A. Shukla, M. Mathew, S.R. Vadera, N. Kumar, J. Lumin. 132, 2023 (2012).

[17] T.A. Pomelova, V.V. Bakovets, I.V. Korol'kov, O.V. Antonova, I.P. Dolgovesova, Phys. Solid State 56, 2496 (2014).

[18] W.-N. Wang, W. Widiyastuti, T. Ogi, I. W. Lenggoro, K. Okuyama, Chem. Mater. 19, 1723 (2007).

[19] O.M. Bordun, I.V. Dovga, I.Yo. Kukharskyy, J. Appl. Spectrosc. 78, 605 (2011). 